\title{
Improving Simplification Performance using FSA : Experimental Result
}

\author{
Nur Fatin Liyana Mohd Rosely', Azlan Mohd Zain ${ }^{1 *}$ and Abdullah Hisham Omar ${ }^{2}$ \\ 'Faculty of Computing, Universiti Teknologi Malaysia, Skudai 81310, Johor, Malaysia; yanarosely@gmail.com, \\ azlanmz@utm.my, \\ ${ }^{2}$ Faculty of Geoinformation and Real Estate, Universiti Teknologi Malaysia, Skudai 81310 Johor, Malaysia; \\ abdullahhisham@utm.my
}

\begin{abstract}
Simplification is generally eliminating unnecessary characteristics of the object without distortion the original shape of the object. Tolerance value is an important parameter needs to be considered produce a good result of simplification process. The current model of simplification has difficulty to execute simplification process in a short time and unable to determine the best tolerance value for simplification process. Fish Swarm Algorithm (FSA) one of the swarm intelligent algorithms with capability of estimating optimum solution shortly was executed in standard simplification design namely FSASimplification. Besides to improve performance of standard simplification, FSA-Simplification is proposed to determine the most optimum and best quality of tolerance value for simplification process. Therefore, this paper carries out the experimental for both simplification models. The roads in Miri, Sarawak with scale ratio 1:1000 is used for simplification process and be displayed in polyline data type. The result shows FSA-simplification has reduced the computing time by $9 \%$ till $12 \%$ that leads to a better simplification result compared to standard simplification. This performance indicates FSA-Simplification has given a better quality of simplification result. Besides that, 10 and 20 are the optimum value and best choice of tolerance value for simplification process. This performance indicates FSA-Simplification has given a better quality of simplification result.
\end{abstract}

Keywords: Cartography Generalization, Fish Swarm Algorithm, Map Object, Simplification Process, Tolerance Value

\section{Introduction}

The process of map data manipulation is named as cartography generalization. In the cartographic generalization process, the essential characteristics or behaviors of objects from map are preserved and they are represented in various scale based on cartographic specifications. For that purposed cartographic generalization offered simplification $\frac{1,15,18}{}$. Simplification helps removing unnecessary detail of object without destroying its essential characteristics ${ }^{13}$. In simplification process, tolerance value is an important parameter need to be considered. The different values of tolerance value reflect different results of simplification process. Others, process time for a simplification process has to be done in short time. According to previous studies by researchers, the main focus only on simplification outcome and slightly ignore the importance of tolerance value. The computing time for a simplification process is also neglected. Therefore, optimization method is the best solution to produce simplification result in short time and to consider the importance of tolerance value in simplification process.

Optimization technique is a technique used in almost fields to handle optimization problem. Generally, there are a lot of optimization techniques have been developed. Recent, implementation of swarm intelligence in optimization algorithms captured the researchers' interest. Among of them, Fish Swarm Algorithm (FSA) has the highest rank for the most convince in handling optimization problem $\underline{2,3,6,7,10,12,23}$. FSA is a swarm intelligence

*Author for correspondence 
evolutionary computation technique proposed by Dr. Xiaolie Li inspired by the natural schooling of fish which has higher plotting time. Hence in this research, FSA is explored to improve simplification process by concerned the process time and takes care of the importance of tolerance value. Based on previous studies, no attempt yet made on FSA in cartographic generalization for object map representation. Therefore this research is considered as a new contribution in it.

\section{Simplification Overview}

Simplification is one of the methods used in cartography generalization to simplify the original object's shape for object map representative in the certain scale. Generally, simplification works by eliminating unnecessary characteristics of an object without distortion the original shape of the object $\mathrm{t}^{19}$. Curved line is an example of the unnecessary characteristics in simplification method. Besides selecting the points which also indicate characteristics of an object, simplification processes are also included to determine point either to retain or reject, remove glitches and reject the redundant points. Then, the best result of the object without destroying its essential shape can be displayed 9 (refer Figure 1). In simplification process, the original characteristics of the object such as orientation, convexity and structure are preserved $\underline{14}$.

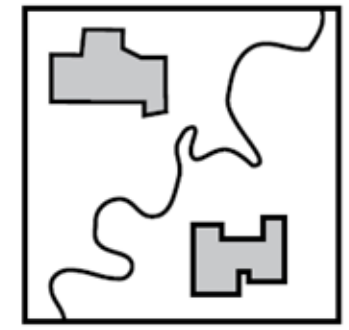

Before simplification process

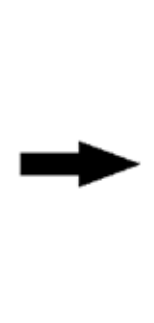

After simplification process
Figure 1. Simplification method.

Based on Figure 1, some characteristics or points of the objects are eliminated during the simplification process. As an example before the simplification process, the representation one of the objects is shown in Figure 1(image Figure 1). During the simplification process, the curve lines of the object that have been circled (image Figure 1) are rejected or eliminated points because they are unnecessary characteristics of the object, (image Figure 1). After the simplification process the object comes out like this (image Figure 1). Further discussion about the simplification process is discussed on the next section.

Besides that, simplification method has ability to tolerate with both data types which are raster data and vector data. However, it most likely suit with the vector data because the presentations of the object map using simplification method are usually displayed in line or polygon ${ }^{11}$. In simplification, line presentation also known as polyline. Generally, as the fundamental unit of the vector data model, $\mathrm{XY}$ coordinate system is applied. The XY coordinates are referring to points of object or also as object characteristics ${ }^{1}$ that indicate latitude and longitude of the object. Based on it, the most suitable XY coordinates should be selected to serve best result from the vector data model.

\section{Simplification Models}

In this study, there are two approach used for simplification process. They are standard simplification and FSASimplification. Both of them are developed in MATLAB environment. Standard simplification has be improved by able to calculate percentage total length reduced for a simplification process, able to define the total number coordinate points of map objects before and after simplification process and finally display two different layout of simplification for a simplification process. Figure 2 illustrates standard simplification flowchart. Meanwhile, FSA-Simplification is developed by enhancing the standard simplification which unable to determine suitable tolerance value for a simplification process. Figure 4 illustrates the flowchart of FSA-Simplification.

\subsection{Standard Simplification}

As mentioned earlier the main purpose of simplification process is to simplify the original shape of map object without eliminate the essential characteristics of the object. In mapping, the smaller scale maps have more map object characteristics to be simplified compared to large scale maps. This happens because small scale maps simply exhibit more area ${ }^{4,5,14,20}$. The simplification process also affected by tolerance value where the smaller value of tolerance value less elimination of map object characteristics and the result also more similar to the original map object characteristics. Based on that, tolerance value is considered as the most important element and without 
it the simplification process won't not happened. Figure 2 illustrates how a simplification process is done using the standard simplification.

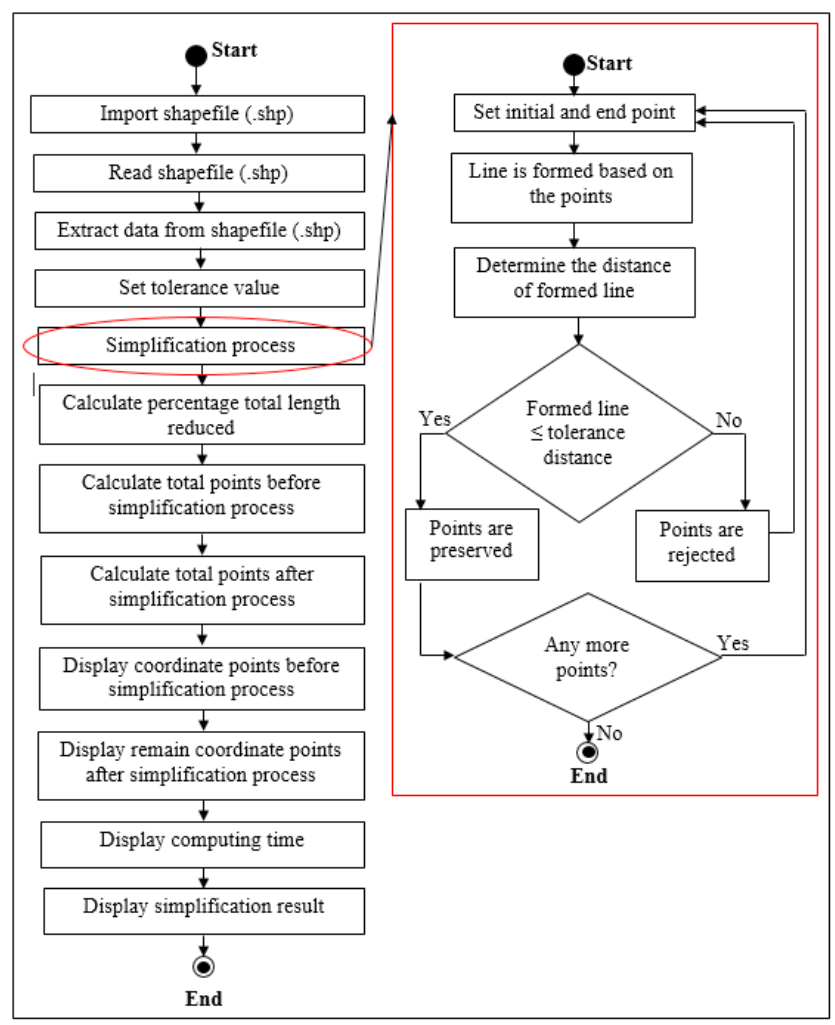

Figure 2. Standard simplification flowchart.

Based on Figure 2, the process starts by import, read and extract data that has been saved as shapefile (.shp) format. Then tolerance value for simplification is set and proceeds with simplification process (refer to circled area). Simplification process begins by deciding initial and end points. Based on these points, a line is formed from the initial point to the end point. Then the formed line is compared to the tolerance value. The formed line is preserved if it does not exceed the tolerance value and the process from assigned points till no more points left is repeated. Otherwise, a new end point is declared and the process continued by forming a new line and compares it to tolerance value. The process is repeated till there no more points left. The process continues by calculating the percentage of total length reduced and total for both coordinate points before and after simplification process. Finally, all needed data are display. The data include coordinate points before and after simplification process, computing time and simplification of map object result. The results of map object simplification are displayed in two different layouts. There are before and after simplification process and overlay result of simplification.

Standard simplification also increases the line crispness as the tolerance value getting higher $\frac{5,8,16}{}$. However, it does not work well with curve line and has slow performances when involve large number of points ${ }^{4}$. Despite on the previous studies, there is not yet research to identify qualities of tolerance value for better result of simplification process. Therefore, optimization technique based on swarm intelligence, Fish Swarm Algorithm (FSA) is implemented for that purposed and named as FSASimplification.

\subsection{FSA-Simplification}

In order to improve the performance of standard simplification, this study proposed FSA-Simplification. The process includes the formulation model of FSA in simplification. The goal of this process is to ensure the modeling of FSA is compatible with behavior of the simplification process. The process flow of current standard simplification and FSA are illustrated in Figure 2 and Figure 3 respectively. Meanwhile, flowchart of the proposed FSASimplification is shown in Figure 4.

\subsubsection{Fish Swarm Algorithm (FSA)}

FSA is inspired by behavior of fish is a swarm intelligence technique and be proposed by Dr. Xiaolie Li in 2002. In water, fish tends to distribute around the area with the high food concentration. FSA is made up of two main components which are parameter and function. The parameter component consists of Step, Visual, crowd factor and food concentration. Meanwhile, search, swarm and follow behaviors are the FSA function component. A fish swarm in a define visual will complete its food foraging by fish behaviors. The movement of fish toward food by among these behaviors is decided by the step size. Inspired by this situation, searching the optimum local and global is created.

As mentioned before, FSA consists of two main components which are parameter and function. Based on the previous studies, the way of parameter define reflect different outputs. As an example, if crowd factor $(\delta)$ has large value, the crowd degree being allowed is smaller and it's better for convergence in the whole area. Meanwhile, the larger the numbers of fish, more distance between fish need to be defined. In FSA, crowd factor and food concentration are related to each other. The fish tend toward 
the area with high food concentration but low crowd factor. This because low crowd factor area means the area not too crowded with others fish so the concentration food available is higher than other place with high crowd factor. However, Step and Visual have greater effect to the result among the FSA parameters. These two parameters must be defined in the early stage and usually they remain constants till the end process. Figure 3 shows how FSA works.

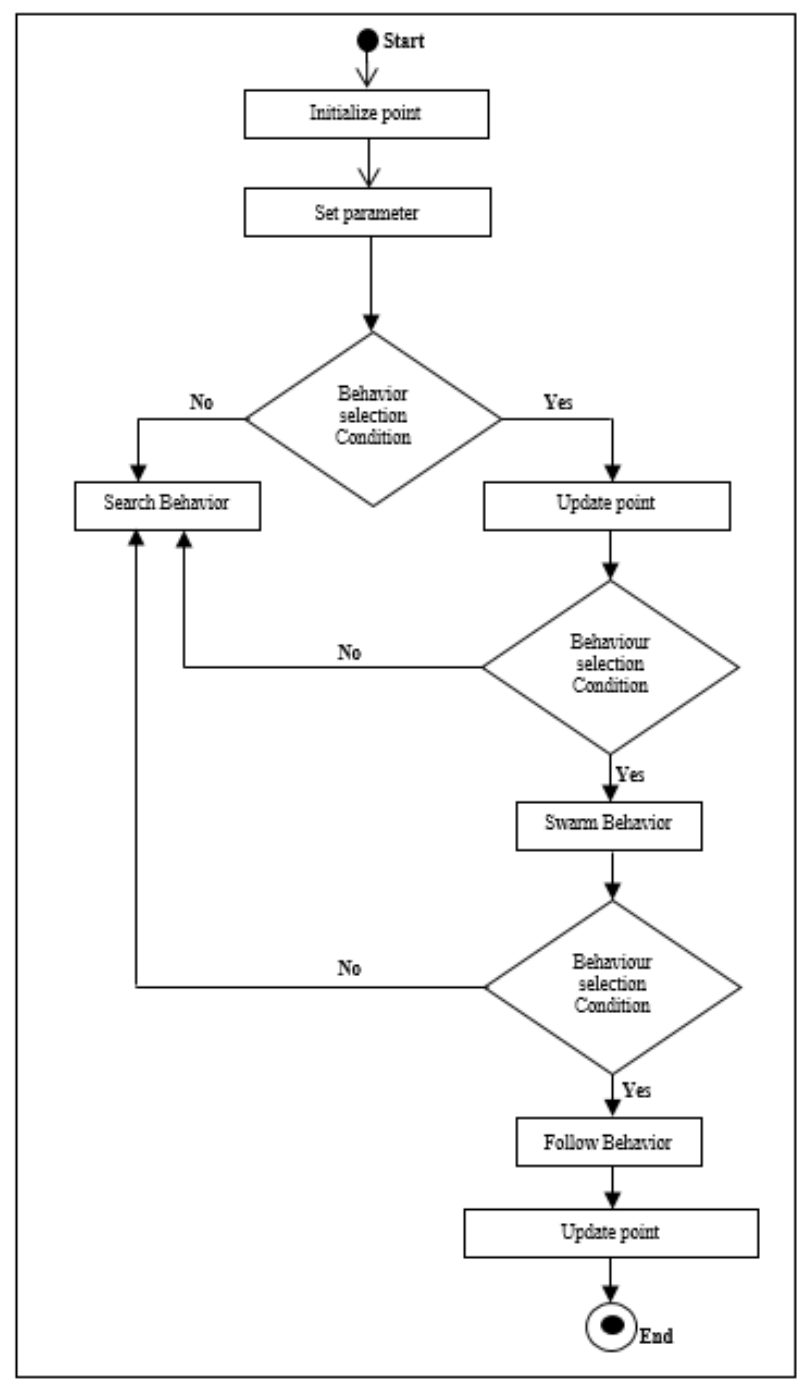

Figure 3. Fish Swarm Algorithm (FSA) flowchart.

Based on Figure 3, the algorithm of FSA is created. The state vector of fish swarm is shown as:

$$
X=\left(X_{1}, X_{2}, X_{3}, \ldots, X_{n}\right)
$$

Where,

$X$ represents the fish and the visual position is presented as:

$$
X_{v}=\left(X_{v 1}, X_{v 2}, X_{v 3}, \ldots, X_{v n}\right)
$$

Where,

$X_{v}$ represent the fish position in Visual.

The above functions (1), (2) worked by:

$$
X_{v i}=X_{v i}+\text { Visual } \times \text { Rand () ; i } \in(0,1]
$$

Where,

$X_{v i}$ is the condition of fish in Visual.

$$
X_{n e x t}=X+\frac{X_{v}-X}{\left(|| X_{v}-X||\right)} \times \operatorname{Step} \times \operatorname{Rand}(O
$$

Where,

$X_{\text {next }}$ is the next fish in Visual.

Based on the Equations (1) to (4), the equations refer to FSA parameters. The behaviors of fish which represent the functions in the FSA only proceed after all above equations are executed. The next step continues with the behavior selection. Equations (5) to (9) represents how the FSA functions worked.

$$
\begin{aligned}
& X_{i}=X_{i}+\text { Visual } \times \text { Rand }(O \\
& \mathrm{x}_{1}=\mathrm{x}_{1}+\text { Visual } \times \text { Rand }(\text { ) } ;
\end{aligned}
$$

- Search Behavior

Where,

If $Y_{i}<Y_{j}$ then it (6) is executed.

$$
X_{i}^{(t+1)}=X_{i}^{(t)}+\frac{X_{i}-X_{i}^{(t)}}{|| X_{i}-X_{i}^{(t)}||} \times \text { Step } \times \text { Rand } O
$$

Else

Execute the (5) by randomly select a state $\mathrm{X}_{\mathrm{j}}$ and test the result by using (6). If still not satisfy even after try number, $t(t<$ Search Behavior) then it moves a step randomly which make it flee from the local extreme value field (7).

$$
\begin{gathered}
X_{i}^{(t+1)}=X_{i}^{(t)}+\text { Visual } \times \text { Rand } 0 \\
X_{i}^{(t+1)}=X_{i}^{(t)}+\frac{X_{j}-X_{i}^{(t)}}{|| X_{c}-X_{c}^{(t)}||} \times \text { Step } \times \text { Rand }(\mathrm{I})
\end{gathered}
$$

$$
\text { - Swarm Behavior }
$$

Execute (8) if satisfied all the follow conditions else executed Search Behavior:

i. The current states of point is $\mathrm{X}_{\mathrm{i}}\left(\mathrm{d}_{\mathrm{i}, \mathrm{j}}<\right.$ Visual $)$ 
ii. $\left(\mathrm{n}_{\mathrm{f}} / \mathrm{n}\right)<\delta$

iii. $\mathrm{Y}_{\mathrm{c}}>\mathrm{Y}_{\mathrm{i}}$

$X_{i}^{(t+1)}=X_{i}^{(t)}+\frac{X_{j}-X_{i}^{(t)}}{|| X_{j}-X_{i}^{(t)}||} \times$ Step $\times$ Rand 0

- Follow Behavior

Execute (9) if satisfied all the follow conditions else executed Search Behavior:

- The current states of point is $\mathrm{X}_{\mathrm{i}}\left(\mathrm{d}_{\mathrm{i}, \mathrm{j}}<\right.$ Visual $)$.

- $\left(\mathrm{n}_{\mathrm{f}} / \mathrm{n}\right)<\delta$.

- $\mathrm{Y}_{\mathrm{j}}>\mathrm{Y}_{\mathrm{i}}$

Based on above Equations, search behavior, swarm behavior and follow behavior are represented as Equations (5), (8) and (9) respectively. They only execute once the related conditions are fulfilled otherwise Equations (6) and (7) are executed. The process will repeat till optimal point is obtained. When the condition is satisfied the current optimal value based on the result obtained is update. Finally, once the termination condition is fulfilled, then the final result is recorded.

As the workflow of FSA shown both components worked well to each others, FSA becomes a hot research topic in many study fields $\frac{2,3,7,21-23}{2}$. Plus, FSA is a flexible swarm intelligence algorithm that able produce high accuracy for optimization output and a fault tolerance $\frac{17,22}{}$. Based on these advantages, FSA is implemented in the simplification process. Next section discussed about how the FSA is implemented and it worked in the simplification process.

\subsubsection{FSA-Simplification Development}

FSA-Simplification is an improved of the standard simplification model that applied FSA in it. The main proposed the implementation is to figure the quality of tolerance value and how it affected the simplification result. Besides, FSA-Simplification also helped in improving the simplification process performance. Figure 4 shows the implementation areas of FSA in the standard simplification model.

Based on Figure 4, FSA-Simplification starts by extract data from shapefile. The points (also known as coordinates) of map object representative are extracted. The values for Visual and Step are also defined in the early process. The Visual and Step in FSA-Simplification repre- sent total number of points for map object representative from shapefile and tolerance value for a simplification process respectively. These values of Visual and Step are also used to calculate coward factor. Then set try number for simplification process. The number of executions of simplification process is determined by this value. The process time (computing time) for each simplification process within the try number is calculated and final average executes time also calculated.

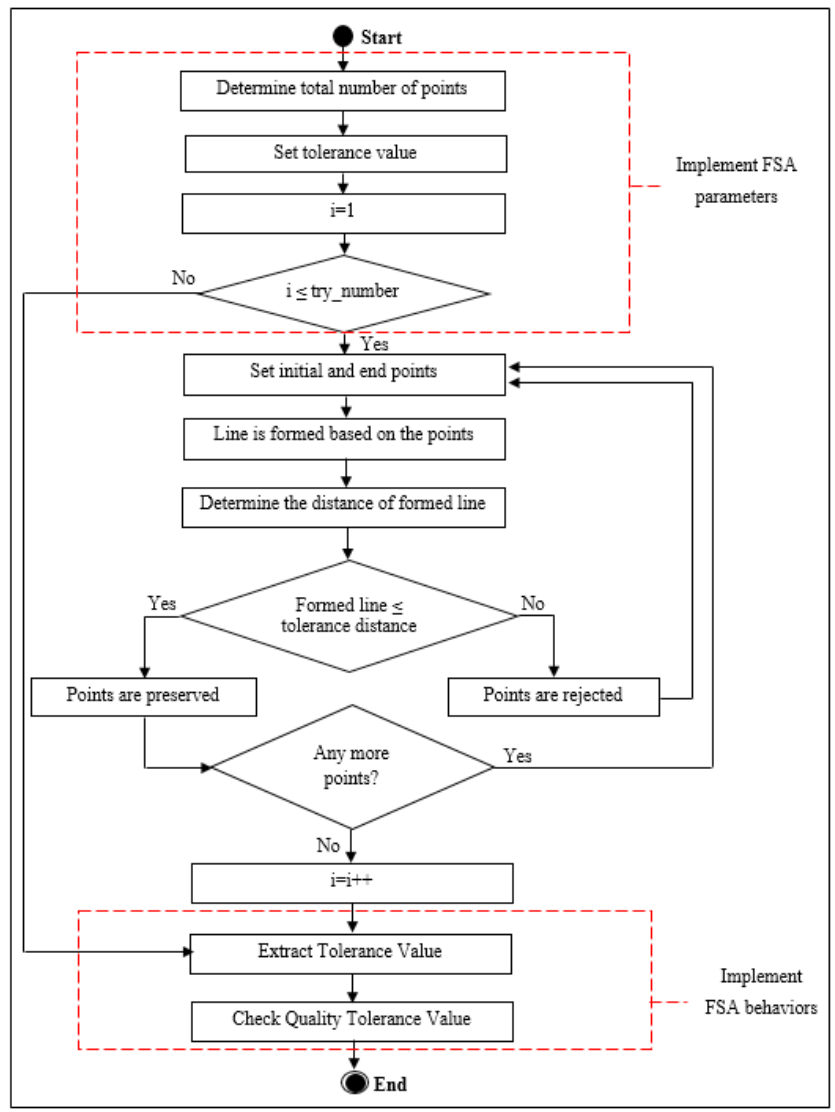

Figure 4. FSA-Simplification flowchart.

The process continued by evaluating the quality of tolerance value either optimum or not for a simplification process. The tolerance value is extracted from the simplification process and be evaluated by using fish behaviors approach. First, the tolerance value is tested with search behavior. The tolerance value is considered as the best optimum value if the Step size is less than coward factor and smaller than or equal to the minimum step for a fish movement. However, if it not fulfilled the conditions, the tolerance value is tested with swarm behavior. In swarm behavior, the tolerance value is tested by comparing with 
value of coward factor and the value between the minimum and maximum size of step for fish movement. The value of tolerance is consider good optimum if satisfied the swarm conditions otherwise the tolerance value either average in or bad quality selection for a simplification process. The tolerance value is consider as average in quality if it satisfied condition for follow behavior and bad value selection for a simplification process if it failed all the behaviors conditions. Finally, update the tolerance quality, the average of computing time, Visual size after simplification process and results of simplification process are displayed.

\section{Experimental Results and Validation}

Experimental results of simplification process are divided into three parts. First, total number coordinates changes after simplification process. Second, results of tolerance computing time. Lastly, result of quality tolerance either it suitable for simplification process or not. First and second result parts involved both simplification models. Meanwhile, last result part only involved FSASimplification. In order to proof the FSA-Simplification helps improved the performance standard simplification for simplification process, computing time for both of them are compared to each other. The differences of computing time between standard simplification and FSA-Simplification based on different tolerance value are measured.

\subsection{Simplification Process}

This section focuses on the result of simplification process using both methods. Roads in the Miri, Sarawak are data set used in this experiment process. Other words, the roads are symbolic to the map object representative in this experiment. Meanwhile, 10, 20, 30, 40, 50, 60, 70, 80,90 and 100 are tolerance value used for both models in simplification process.

Based on the Figure 5, the roads in Miri, Sarawak is displayed in polyline data type with ratio 1:1000. The data is shapefile (.shp) format. The vector data of roads are represented in coordinates and be displayed as polyline data type. The coordinates reflect latitude and longitude of roads. The latitude and longitude are also known as $\mathrm{x}$-coordinates and $\mathrm{y}$-coordinates respectively $\underline{\underline{13}}$. The source of data is from Jabatan Ukur dan Pemetaan Malaysia (JUPEM).

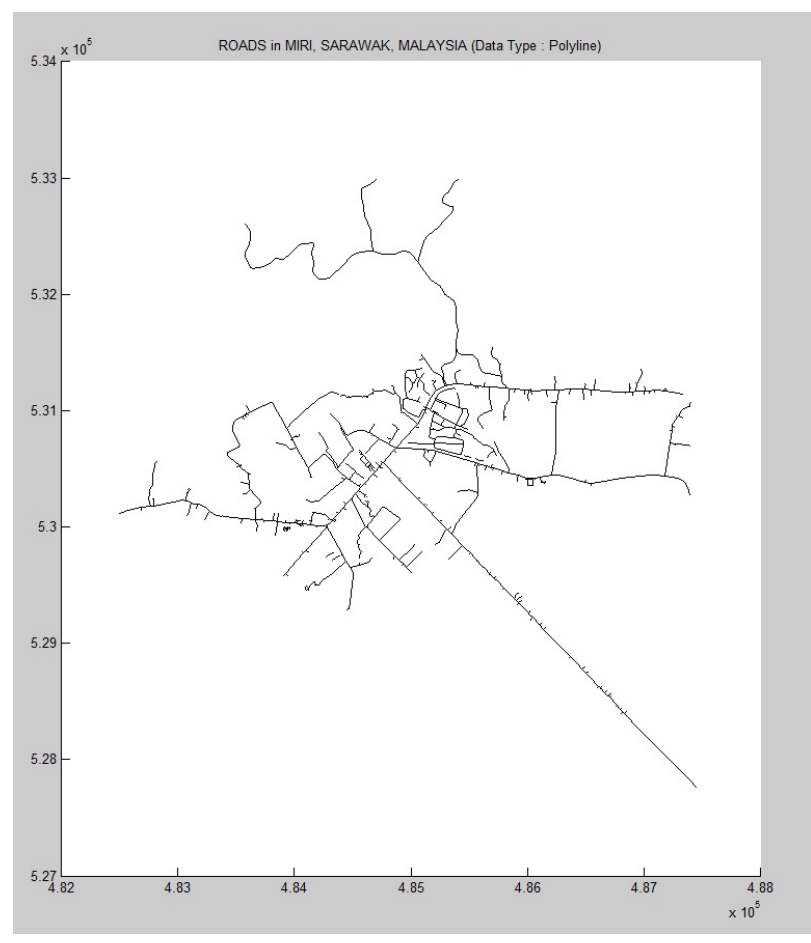

Figure 5. Roads in Miri, Sarawak.

The total numbers of coordinates before simplification process are similar but differ after simplification process. The results of simplification process are different because tolerance value play huge role in determine the outcome of the simplification process. Therefore, the numbers of map object points that represent the coordinates after the simplification process are also differing to each other. Table 1 also shows the numbers of points based on total pair of coordinates before and after simplification process (Refer columns; Total coordinate in pairs).

Table 1. Total coordinates of simplification process

\begin{tabular}{|l|l|l|}
\hline \multirow{2}{*}{$\begin{array}{l}\text { Tolerance } \\
\text { Value (TV) }\end{array}$} & \multicolumn{2}{|l|}{ Total coordinate in pairs } \\
\cline { 2 - 3 } & $\begin{array}{l}\text { Original Map } \\
\text { Object Coordinates }\end{array}$ & $\begin{array}{l}\text { Simplified Map } \\
\text { Object Coordinates }\end{array}$ \\
\hline 10 & 2870 & 2021 \\
\hline 20 & 2870 & 1944 \\
\hline 30 & 2870 & 1918 \\
\hline 40 & 2870 & 1904 \\
\hline 50 & 2870 & 1899 \\
\hline 60 & 2870 & 1888 \\
\hline 70 & 2870 & 1886 \\
\hline 80 & 2870 & 1883 \\
\hline 90 & 2870 & 1878 \\
\hline 100 & 2870 & 1878 \\
\hline
\end{tabular}


Referring to Table 1 , the higher tolerance value, the smaller total number of coordinates in pairs after simplification process. This situation also indicates essential points of map object might be rejected. Figure 6 to Figure 9 show several result of simplification with different tolerance value. Several areas are assigned as a, b, c, d and e to reflect and show obvious effect of simplification process. However, there is condition where unsuitable selection of tolerance value may affect the result of simplification process by totally change the shape of map objects. As example, the result simplification with tolerance values, 60,00 and 100.00 shown huge changes of original map objects especially at a, $c$ and e circled areas. As simplification process should prevent the essential points from be rejected, a wise selection of tolerance value is needed. Hence, FSA-Simplification is proposed.

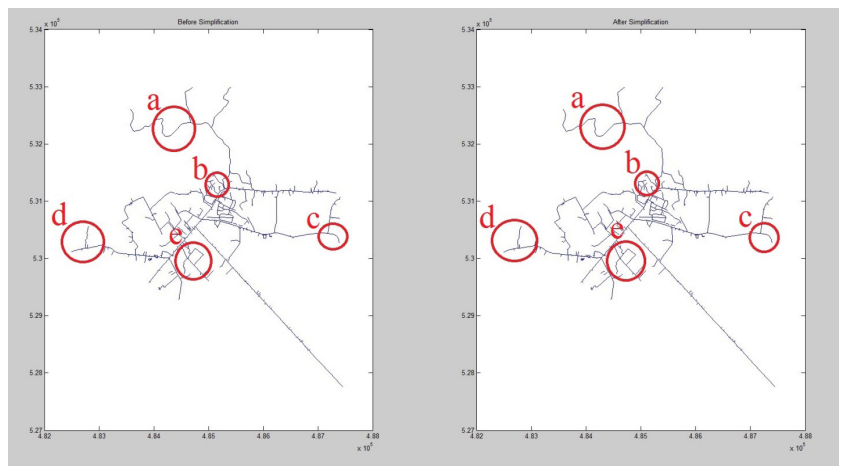

Figure 6. Before and after simplification, TV $=10.00$.

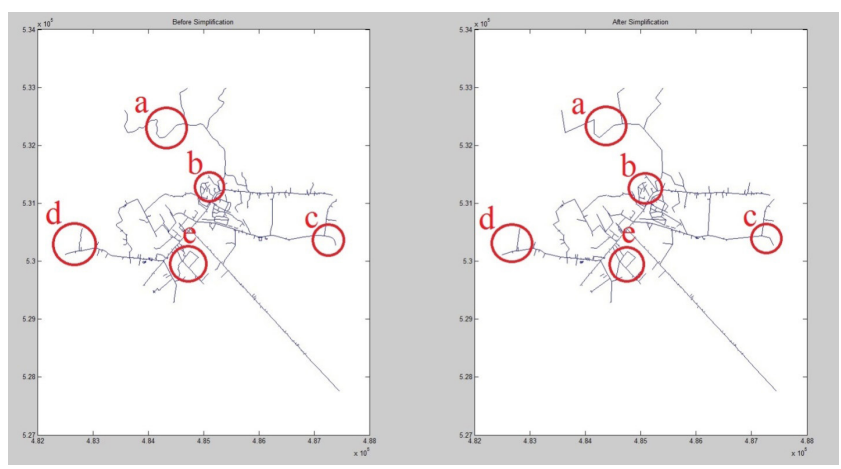

Figure 7. Before and after simplification, TV $=50.00$.

\subsection{Tolerance Computing Time}

In this study, total experiment set for standard simplification is 10 sets which each set involved 10 runs times. Therefore, total experiments are 100 runs times. Meanwhile, total experiment set for FSA-Simplification is 10 sets where each set involved 10 runs times. Therefore, total experiments are 100 runs times. However, by implementing try number, each set already perform 10 runs times in an experiment execution. As the result every experiment execution gives average computing time for 10 run times. This means for 100 run times only 10 experiment executions are needed. Seconds (s) is measurement for computing time.

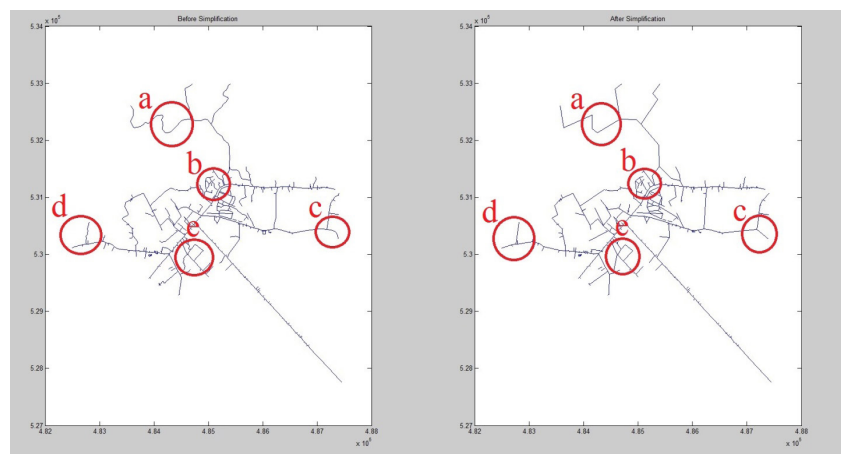

Figure 8. Before and after simplification, TV $=60.00$.

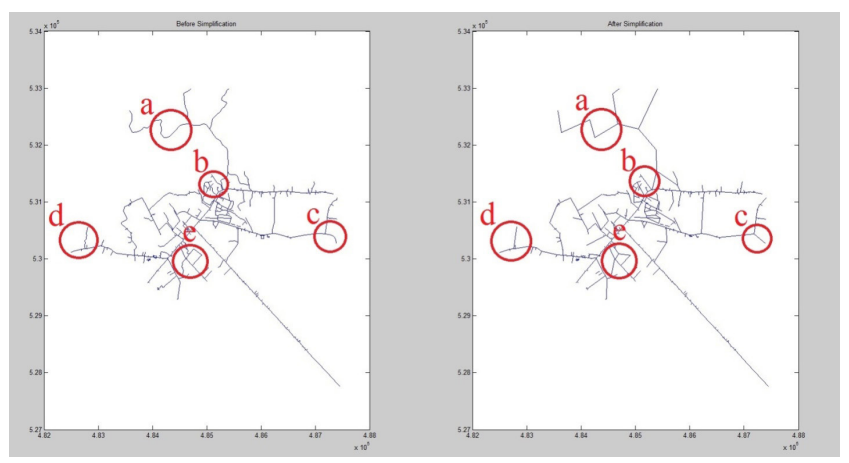

Figure 9. Before and after simplification, TV $=100.00$.

Table 2 and Table 3 record the final computing time of both models with different tolerance value. Analysis for both models performance for simplification process is recorded as Table 4.

Table 2. Average computing time of standard simplification with different tolerance value

\begin{tabular}{|l|l|}
\hline Tolerance Value (TV) & Average Time (s) \\
\hline 10 & 0.573813 \\
\hline 20 & 0.560009 \\
\hline 30 & 0.555856 \\
\hline 40 & 0.553047 \\
\hline 50 & 0.552402 \\
\hline 60 & 0.551827 \\
\hline 70 & 0.549128 \\
\hline
\end{tabular}




\begin{tabular}{|l|l|}
\hline 80 & 0.548705 \\
\hline 90 & 0.546430 \\
\hline 100 & 0.545278 \\
\hline
\end{tabular}

Based on Table 2, it is the final result of standard simplification for a simplification process with different tolerance value. The final computing time is based on the average computing time for 100 runs times of experiments. The computing times for each tolerance value are different. The higher tolerance value the less computing time taken. As example, TV $=10$ the computing time is 0.573813 s meanwhile $\mathrm{TV}=100$ the computing time is 0.545278 s. Figure 10 reflect result from Table 2.

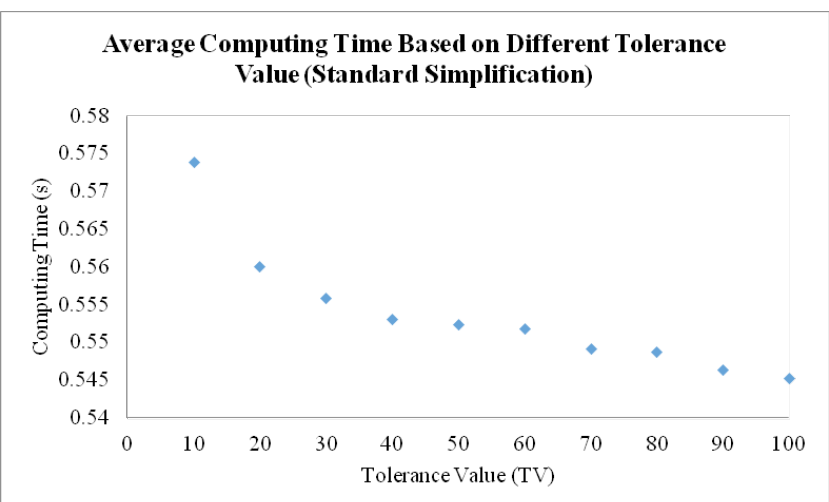

Figure 10. Average computing time of standard simplification with different tolerance value.

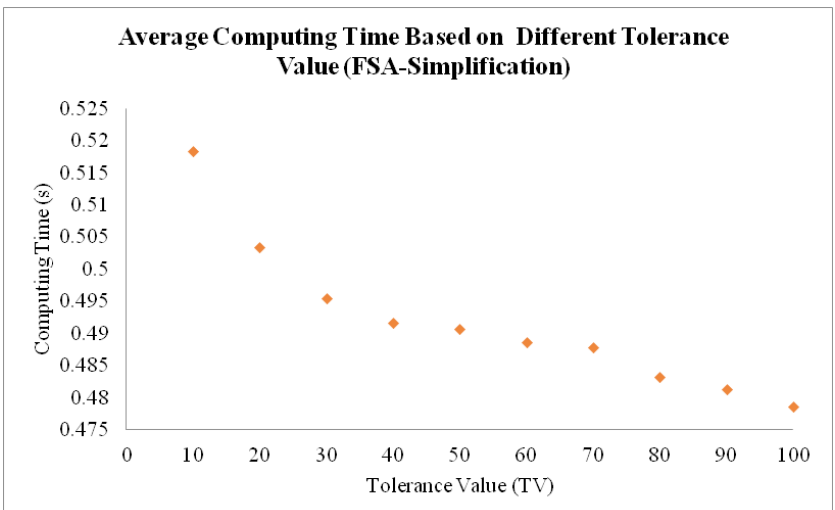

Figure 11. Average computing time of FSA-Simplification with different tolerance value.

Based on Figure 10, the average computing time for simplification decreases as the tolerance value gets higher. This means, less computing time taken, more points of map objects are eliminated during simplification process. This situation may lead to elimination of essential points so by defining the quality of tolerance value for a simplification is a good alternative to prevent elimination of essential points of map objects. For that purpose FSASimplification is introduced. Table 3 and Figure 11 show simplification results by using FSA-Simplification.

Table 3. Computing time of FSA-Simplification with different tolerance value

\begin{tabular}{|l|l|}
\hline Tolerance Value (TV) & Average Time (s) \\
\hline 10 & 0.51833 \\
\hline 20 & 0.50343 \\
\hline 30 & 0.49545 \\
\hline 40 & 0.49159 \\
\hline 50 & 0.49066 \\
\hline 60 & 0.48865 \\
\hline 70 & 0.48777 \\
\hline 80 & 0.48322 \\
\hline 90 & 0.48134 \\
\hline 100 & 0.47853 \\
\hline
\end{tabular}

Based on Table 3, it is the final result of FSASimplification for a simplification process with different tolerance value. The final computing time is based on the average computing time for 100 runs times of experiments. The computing times for each tolerance value are different. The smaller tolerance value more computing time is taken. As example, TV $=10$ the computing time is $0.51833 \mathrm{~s}$. However, TV $=100$ the computing time is $0.47853 \mathrm{~s}$. Meanwhile, Figure 11 reflects the result in Table 3 in graph illustration.

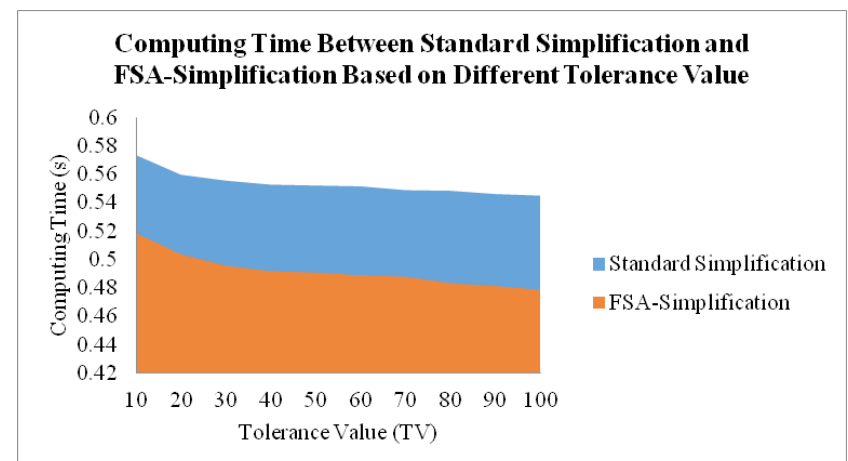

Figure 12. Computing time between standard simplification and FSA-Simplification based on different tolerance value.

Based on Table 4, the performance for both standard simplification and FSA-Simplification are listed based on 
tolerance value used in simplification process. The differences of average computing time between both models are calculated (refer to difference of computing time column). Based on that, percentage of difference and percentage of performance improved from standard simplification to FSA-Simplification (refer to percentage of improved performance column) are measured.

Table 4. Analysis result between standard simplification and FSA-Simplification

\begin{tabular}{|l|l|l|l|}
\hline $\begin{array}{l}\text { Tolerance } \\
\text { Value } \\
(\mathrm{TV})\end{array}$ & $\begin{array}{l}\text { Performance } \\
\text { Difference } \\
(\mathrm{s})\end{array}$ & $\begin{array}{l}\text { Percentage } \\
\text { of differences } \\
(\%)\end{array}$ & $\begin{array}{l}\text { Percentage } \\
\text { the improve } \\
\text { performance } \\
(\%)\end{array}$ \\
\hline 10 & 0.055483 & 5.548300 & 9.669178 \\
\hline 20 & 0.056579 & 5.657885 & 10.10321 \\
\hline 30 & 0.060406 & 6.040623 & 10.86724 \\
\hline 40 & 0.061457 & 6.145685 & 11.11241 \\
\hline 50 & 0.061742 & 6.174169 & 11.17696 \\
\hline 60 & 0.063177 & 6.317681 & 11.44867 \\
\hline 70 & 0.061358 & 6.135801 & 11.17372 \\
\hline 80 & 0.065485 & 6.548487 & 11.93444 \\
\hline 90 & 0.06509 & 6.508995 & 11.91186 \\
\hline 100 & 0.066748 & 6.674772 & 12.24105 \\
\hline
\end{tabular}

Referring to average computing time, both models show a smaller tolerance value, higher computing time. The difference of computing time based on tolerance value increase as the tolerance value increased. This automatically indicates the percentage of difference also increased corresponding to the increment of tolerance value. As the final result, FSA-Simplification is capable to improve the simplification performance from $9.67 \%$ to $12.241 \%$.

Figure 12 plots the computing time between standard simplification and FSA-Simplification based on different tolerance value together. The figure shows standard simplification took longer computing time compared to FSA-Simplification. Figure 13 shows the percentage improvement of simplification performance from standard simplification to FSA-Simplification.

\subsection{Quality Tolerance}

Quality tolerance for a simplification process is measured using the proposed FSA-Simplification. 'BEST', 'GOOD', 'AVERAGE', 'BAD' are represent the quality of tolerance value. Table 5 records the quality of tolerance for each tolerance value used for this study.

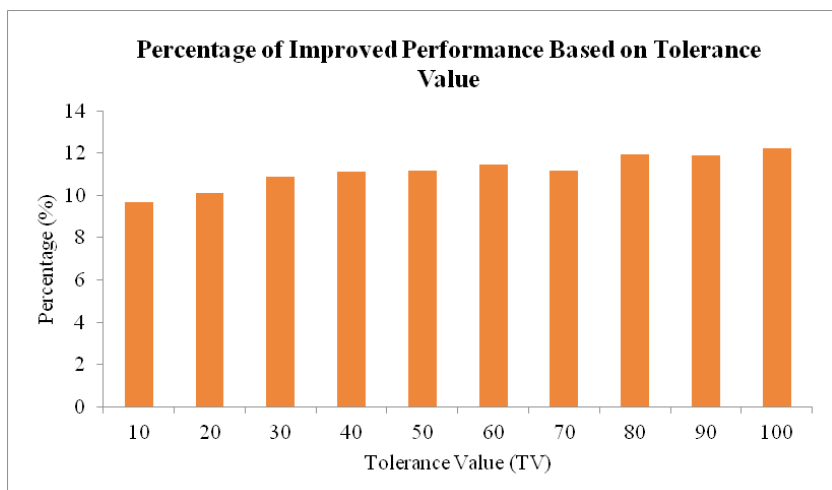

Figure 13. Percentage of improved performance based on tolerance value.

Table 5. Quality of each tolerance value

\begin{tabular}{|l|l|}
\hline Tolerance Value (TV) & Quality of TV \\
\hline 10 & Best \\
\hline 20 & Best \\
\hline 30 & Good \\
\hline 40 & Good \\
\hline 50 & Good \\
\hline 60 & Average \\
\hline 70 & Bad \\
\hline 80 & Bad \\
\hline 90 & Bad \\
\hline 100 & Bad \\
\hline
\end{tabular}

Based on Table 5, 10 and 20 are considered as best tolerance value. Both values passed first condition, search behavior. The condition is tested whether the tolerance value has high ability to preserve maximum essential points of map object after simplification process. This reflects the simplified map object with 10 and 20 as tolerance values able to preserved most of the essential points. 3040 and 50 are good consideration for tolerance value selection. These three failed the search behavior but passed swarm behavior. This situation reflects some essential points of map object may not be preserved. However, the simplified map objects not violent the original essential points of map object and it can be good tolerance approach for small scale maps. This because small scale maps have more points of map object to be simplified ${ }^{14,20}$. 60 is average quality of tolerance value where it passed follow behavior but failed others two behavior. This reflect essential points of map objects are rejected and may cause violent to original map object and it not recommended for simplification process. 70, 80, 90 and 100 
are bad choice of tolerance value as they failed all behaviors condition. This reflect most essential points of map objects are rejected and simplified map object violent the original map object. As result these tolerance value highly not recommended for simplification process.

\section{Conclusion}

Standard simplification can be considered as good practice for simplification process. However, standard simplification could not able to trace the optimum and best tolerance value for a simplification process. This situation quite worrisome because tolerance value plays important role to determine either to preserve or eliminate map objects characteristics or points. Therefore, FSA-Simplification is proposed to overcome the problem based on experimental result, besides 10 and 20 are declared as the optimum and best tolerance value for simplification process. FSA-Simplification also shows improvement in computing time. The percentage difference of computing time between standard simplification and FSA-Simplification is from $5.548 \%$ to $6.675 \%$. This also indicates the performance improve from $9.670 \%$ to $12.241 \%$.

\section{Recommendations}

This study has implemented FSA in standard simplification to improve the computing time and to determine the best tolerance for a polyline simplification process. Basically, FSA consists of two main components which are parameter and function. For future work, FSA can be considered to be implemented in other polyline simplification. The implementations of FSA can be either in Fast Total Least Square (FTLS), Semantically Enriched Line Simplification (SELF), Douglas Peucker Algorithm (DPA) or others. However, for the purpose on improving the limitation of FSA-Simplification, FSA in DPA is recommended. Since the strategy of implementation introduced in this study is flexible, there would be no problem to apply the recommended technique.

\section{Acknowledgement}

Special appreciation to reviewers for useful advices and comments. The authors greatly acknowledge the Research Management Centre, UTM and Ministry of Higher Education Malaysia (MOHE) for financial support through the Fundamental Research GrantScheme GUP TIER1/ Q.J130000.2528.14H13s

\section{References}

1. Burrough PA, McDonnell RA. Principles of geographical information systems. Oxford University Press; 2011. 19988.

2. El-said SA. Image quantization using improved artificial fish swarm algorithm. Soft Computing; 2014. p. 1-13.

3. Fei W, Xiao-Hao X, Jing Z. Application of artificial fish school and K-means clustering algorithms for stochastic GHP. IEEE Control and Decision Conference, CCDC'09; 2009 Jun. p. 4280-3.

4. Gao P, Liu Z, Han F, Tang L, Xie M. Accelerating the computation of multi-scale visual curvature for simplifying a large set of polylines with Hadoop. GIScience and Remote Sensing. 2015; 52(3):315-31.

5. Hu T, Ren XY, Lu YM. Simplified calculation of distance measure in DP algorithm. Fifth International Conference on Graphic and Image Processing; 2014 Jan. p. 906921.

6. Huang Z, Chen Y. An improved artificial Fish Swarm Algorithm based on hybrid behavior selection. International Journal of Control and Automation. 2013; 6(5):103-16.

7. Huang ZJ, Wang BQ. A novel swarm clustering algorithm and its application for CBR retrieval. 2nd IEEE International Conference on Information Engineering and Computer Science (ICIECS); 2010 Dec. p. 1-5.

8. Jelinek A, Zalud L, Jilek T. Fast total least squares vectorization. Journal of Real-Time Image Processing. 2016; 1-17.

9. Lee D. Automation of map generalization: The cutting-edge technology. Esri White Paper Series; 1996.

10. Liu S, Li Y. An artificial Fish Swarm Algorithm and its application. 2015 International Conference on Intelligent System Research and Mechatronics Engineering; Atlantis Press. 2015 Apr.

11. McMaster RB. Automated line generalization. Cartographica: The International Journal for Geographic Information and Geovisualization. 1987; 24(2):74-111.

12. Neshat M, Sepidnam G, Sargolzaei M, Tosi AN. Artificial Fish Swarm Algorithm: A survey of the state-of-the-art, hybridization, combinatorial and indicative applications. Artificial Intelligence Review; 2012. p. 1-33.

13. Raheja JL, Kumar U, Saravanan KC. Cartographic generalization (Selection) for 2D map of urban area. International Journal of Computer Science and Information Technology. 2010;2(1). 
14. Sester M. Generalization based on least squares adjustment. International Archives of Photogrammetry and Remote Sensing. 33(B4/3; PART 4). 2000; 931-8.

15. Shea KS, McMaster RB. Cartographic generalization in a digital environment: When and how to generalize. Proceedings of AutoCarto. 1989 Apr; 9:56-67.

16. Stefanakis E. SELF: Semantically Enriched Line Simplification. International Journal of Geographical Information Science. 2015; 29(10):1826-44.

17. Tao L, Ai-ling Q, Yuan-Bin H, Xin-Tan C. Feature optimization based on artificial Fish Swarm Algorithm in intrusion detections. IEEE International Conference on Networks Security, Wireless Communications and Trusted Computing, NSWCTC'09; 2009 Apr; 1:542-5.

18. Vangenot C, Parent C, Spaccapietra S. Modelling and manipulating multiple representations of spatial data. Advances in Spatial Data Handling. Springer Berlin Heidelberg; 2002. p. 81-93.
19. Weibel R. Generalization of spatial data: Principles and selected algorithms. Algorithmic Foundations of Geographic Information Systems. Springer Berlin Heidelberg; 1997. p. 99-152.

20. Wu ST, Marquez MRG. A non-self-intersection DouglasPeucker algorithm. IEEE XVI Brazilian Symposium on Computer Graphics and Image Processing, SIBGRAPI; 2003, Oct. p. 60-6.

21. Yazdani D, Nabizadeh H, Kosari EM, Toosi AN. Color quantization using modified artificial Fish Swarm Algorithm. AI 2011: Advances in Artificial Intelligence. Springer Berlin Heidelberg; 2011. p. 382-91.

22. Yiyue W, Hongmei L, Hengyang H. Wireless Sensor Network deployment using an optimized artificial Fish Swarm Algorithm. 2012 IEEE International Conference on Computer Science and Electronics Engineering (ICCSEE). 2012 Mar; 2:90-4.

23. Zhang C, Zhang FM, Li F, Wu HS. Improved artificial Fish Swarm Algorithm. 2014 IEEE 9th Conference on Industrial Electronics and Applications (ICIEA); 2014 Jun. p. 748-53. 